\title{
QUEEN'S
UNIVERSITY
BELFAST
}

\section{Millimeter Wave Hybrid Beamforming with Rotman Lens: Performance with Hardware Imperfections}

Abbasi, M. A. B., Fusco, V., \& Matthaiou, M. (2019). Millimeter Wave Hybrid Beamforming with Rotman Lens: Performance with Hardware Imperfections. In 2019 16th International Symposium on Wireless Communication Systems (ISWCS) (pp. 203-207). Institute of Electrical and Electronics Engineers Inc.. https://doi.org/10.1109/ISWCS.2019.8877186

Published in:

2019 16th International Symposium on Wireless Communication Systems (ISWCS)

Document Version:

Peer reviewed version

Queen's University Belfast - Research Portal:

Link to publication record in Queen's University Belfast Research Portal

Publisher rights

Copyright 2019 IEEE. This work is made available online in accordance with the publisher's policies. Please refer to any applicable terms of use of the publisher.

\section{General rights}

Copyright for the publications made accessible via the Queen's University Belfast Research Portal is retained by the author(s) and / or other copyright owners and it is a condition of accessing these publications that users recognise and abide by the legal requirements associated with these rights.

Take down policy

The Research Portal is Queen's institutional repository that provides access to Queen's research output. Every effort has been made to ensure that content in the Research Portal does not infringe any person's rights, or applicable UK laws. If you discover content in the Research Portal that you believe breaches copyright or violates any law, please contact openaccess@qub.ac.uk. 


\title{
Millimeter Wave Hybrid Beamforming with Rotman Lens: Performance with Hardware Imperfections
}

\author{
M. Ali Babar Abbasi, Vincent F. Fusco and Michail Matthaiou \\ Institute of Electronics, Communications and Information Technology (ECIT), Queen's University Belfast, Belfast, U.K. \\ e-mails: \{m.abbasi, v.fusco and m.matthaiou\}@qub.ac.uk
}

\begin{abstract}
A two-stage Rotman lens based millimeter wave (mmWave) hybrid beamforming architecture for multiuser multiple-input multiple-output (MU-MIMO) operation is investigated. We identify a set of non-trivial losses within the complex analog phase-shifter network with the aid of a subscaled beamformer prototype, consisting of 15 element uniform rectangular array (URA) and two stage phase-shifter with 6 stacked Rotman lenses. The prototype is designed to operate at $28 \mathrm{GHz}$ and is capable of 9 beam projections in azimuth and elevation zones. We evaluate the ergodic sum spectral efficiency of a 9-user uplink MU-MIMO system when the user equipments' (UE) dominant paths are assumed to be aligned with the predefined beam directions. We point out the primary sources of some of the most prominent EM losses and discuss design guidelines for their possible mitigation at both the RF front-end and the signal processing (SP) blocks. Our investigation shows the practical limitations of the system and predicts the achievable performance. Interestingly, our conclusions hold valid for a scaled version of the same hybrid beamformer architecture supporting a larger number of antenna elements.
\end{abstract}

\section{INTRODUCTION}

Multiple-input multiple-output (MIMO) communication systems operating at millimeter wave (mmWave) frequencies face an inevitable free space path loss which is generally mitigated by using antenna arrays with high directivity. Focusing electromagnetic energy towards a specific direction is in contrast to commonly used nearly omni-directional antennas at sub-6 GHz frequencies. A standard antenna array is capable of projecting only a single beam in a specific direction, while to provide coverage in a specific sector in a communication system, beam scanning capabilities are added to an array by using a network of phase-shifters. In addition to free space losses at mmWave frequencies, device level losses at the radio frequency (RF) front-ends are much more pronounced as compared to the same at sub-6 GHz frequencies. These losses are primarily due to practical hardware impairments at mmWave frequencies and they strongly impact the system link-budget. Hence, proper quantification of these losses is vital to accurately estimate the theoretical limits of a mmWave MIMO system.

In a conventional MIMO transceiver, the baseband processing unit is normally directly connected to each antenna element. This means a dedicated down-conversion/up-conversion $\mathrm{RF}$-chain (amplifier, filter, mixer etc.) per antenna. One possible solution to this high-cost exercise is hybrid beamforming, where each antenna element is first connected to a RF-front end network, and then to the signal processing (SP) unit
[1]-[5]. Classifications and trade-off factors of such hybrid beamforming architectures were studied in detail in [2]. One of the most important benefits of hybrid beamforming is the possibility of using less number of active RF-chains to support comparatively larger number of antenna elements. To make this happen, almost all the hybrid architectures suggest sharing RF front-end equipment in one way or the other, which, at mmWave frequencies, is not as straightforward as sharing the same array aperture by the antenna elements. To elaborate on this, let us assume a 64 element antenna array connected to $16 \mathrm{RF}$-chains. Theoretically, an $8 \times 8$ element uniform antenna array with isotropic antenna elements is capable of directive beam with half-power beam width (HPBW) of $\sim 13^{\circ}$. To provide coverage to a $\pm 50^{\circ}$ sector along azimuth, at least 8 beam states are required. To achieve this, each antenna element needs to be connected to an 8-state phase shifter which is a cumbersome task. On top of it, MIMO spatial coverage relies very much on the accurate directivity of beams, which requires highly accurate phase shifters. This further increases the cost and complexity of the network. Note that the hardware required for a single beam scanning can only support a single RF-chain and, consequently, a single user equipment (UE) in a multi-user MIMO (MU-MIMO) setup. $16 \mathrm{RF}$-chains supported by the same system at the same time requires either a separate active phase shifter network sharing the same antenna array, or the same phase shifter network and the antenna array with a separate RF-switching block. Some examples of such complex base-station (BS) arrays are [6]-[8]. Carefully designed lens-based analog beamformers provide a lucrative alternative and in this paper we discuss one such example.

More specifically, we discuss a Rotman lens based mmWave beamforming architecture and show the beam projection along azimuth and elevation zones. This technique of simplifying the beamformer hardware does not come for free, in terms of losses at the mmWave RF front-end. We elaborate on these hardware impairments and propose mitigation techniques. The uplink MU-MIMO performance of the same beamformer was previously evaluated in [9]. In this work, we first show that the number of active $\mathrm{RF}$-chains required for mmWave MIMO operation can be less than the number of antenna elements in a URA using a stacked analog phase-shifter topology. The losses associated with this technique can be reduced by engineering strategies. We further evaluate and show the practical spectral efficiency of the Rotman lens based mmWave 


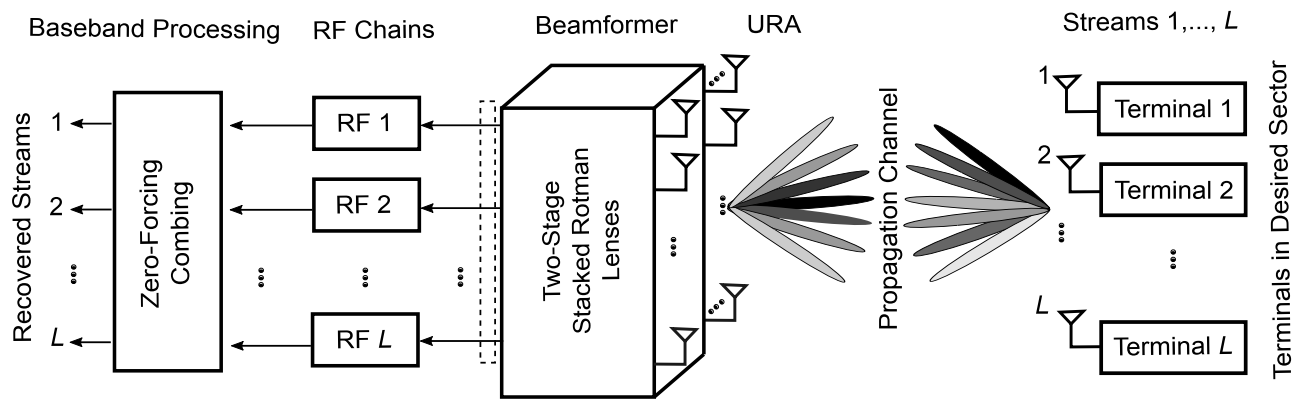

Fig. 1. MU--MIMO uplink hybrid architecture with two-stage Rotman lens based URA beamformer [9].

hybrid architecture involving all the hardware imperfections.

\section{Hybrid BEAMFORMER ARCHITECTURE}

We consider the uplink MU-MIMO system in Fig. 1 where the BS array is equipped with a two-stage Rotman lens-based beamformer, connected to $L$ RF chains and baseband SP unit. We assume uniformly distributed UEs within a $100^{\circ}$ zone in azimuth and $30^{\circ}$ zone in elevation sector. A URA is considered in receive mode when an uplink data stream from $L$ UEs are transmitted at an equal power sharing the same time-frequency resource. We assume a perfect knowledge of the propagation channel at both the BS and UE ends. The coverage zone for the URA beamforming operation was selected to be in line with the prescribed standards of mmWave MIMO. However, it is not possible to cover the entire sector with only 15 element URA, and to simplify the problem, the uplink direction of arrivals (DOAs) are assumed to be aligned with the beam directions. The gain and phase at the output of the beamformer are down converted to $L$ RF chains for baseband SP (Fig. 1). The $L \times 1$ down-converted signal can be written as:

$$
\mathbf{y}=\rho_{\mathrm{t}}^{\frac{1}{2}} \mathbf{F}_{\mathrm{RF}} \mathbf{H x}+\mathbf{n}=\rho_{\mathrm{t}}^{\frac{1}{2}} \mathbf{G} \mathbf{x}+\mathbf{n}
$$

when $\mathbf{G}=\left[\mathbf{g}_{1} \mathbf{g}_{2} \ldots \mathbf{g}_{L}\right]$ is an $L \times L$ matrix when $\mathbf{g}_{\ell}=$ $\mathbf{F}_{\mathrm{RF}} \mathbf{h}_{\ell}, \forall \ell=1,2, \ldots, L$ is $L \times 1$ vector [9]. $\mathbf{F}_{\mathrm{RF}}$ will be defined later in (3). We modeled the $M \times 1$ uplink channel vector for the $\ell$-th terminal as double-directional finite multipath components (MPCs) [10] as:

$$
\mathbf{h}_{\ell}=\frac{1}{\sqrt{N_{\mathrm{P}}}} \sum_{p=1}^{N_{\mathrm{P}}} \xi_{\ell, p} \Lambda\left(\phi_{\ell, p}, \theta_{\ell, p}\right) \mathbf{a}^{H}\left(\phi_{\ell, p}, \theta_{\ell, p}\right),
$$

where $N_{\mathrm{P}}$ is the number of MPCs, $\xi_{\ell, p}$ represents the gains of the $p$-th MPC, $\Lambda\left(\phi_{\ell, p}, \theta_{\ell, p}\right)$ is the per-antenna element gain, and $\mathbf{a}\left(\phi_{\ell, p}, \theta_{\ell, p}\right)$ is the far-field steering vector of the URA.

\section{ROTMAN LENS BASED BEAMFORMER}

We design a BS URA in Fig. 2 using microstrip patch antenna unit cells operating at $28 \mathrm{GHz}$. The antenna unit cell is designed and fabricated on Roger 4003C substrate with electrical properties $\epsilon_{\mathrm{r}}=3.38, \tan (\delta)=0.0027$. The height of the substrate is $813 \mu \mathrm{m}$ while the length and the width of the patch are $2.48 \mathrm{~mm}$ and $2.18 \mathrm{~mm}$ respectively. The antenna elements are excited via coax feed when the coax pin is 0.3 $\mathrm{mm}$ inside the patch from the top edge along the length. The antenna array is operational at the fundamental resonant mode [11] with a $10 \mathrm{~dB}$ bandwidth of $3650 \mathrm{MHz}$ and per-element

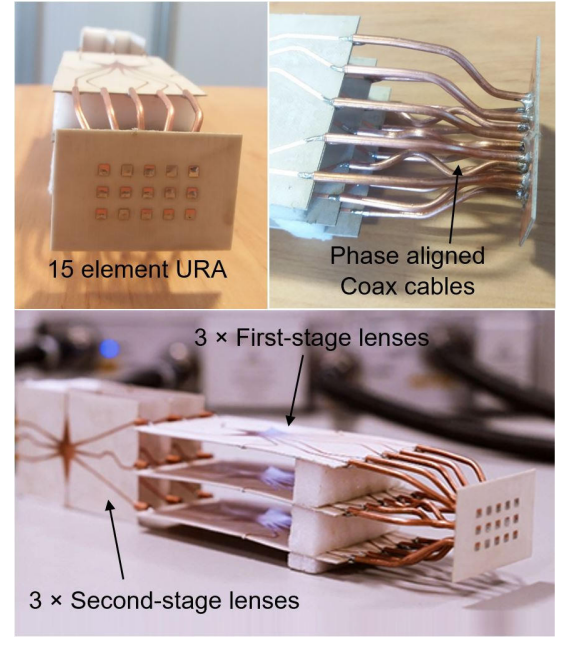

Fig. 2. Two-stage Rotman lens-based beamforming URA prototype.

realized gain of $6.8 \mathrm{dBi}$. The antenna is replicated in 3 rows and 5 columns in a URA formation.

We connect this URA with Rotman lenses via phase aligned coax cables. The first stage lenses are responsible of azimuth coverage and the second stage lenses are responsible of elevation beamforming. The first stage lens has $N_{\text {ap }}=5$ array ports and $N_{\mathrm{bp}}=3$ beam ports, while the second stage lens has $N_{\text {ap }}=3$ and $N_{\text {bp }}=3$. Both stage lenses are fabricated on Taconic-RF 60 substrate with $640 \mu \mathrm{m}$ thickness and having material properties: $\epsilon_{\mathrm{r}}=6.15$, and $\left.\tan (\delta)=0.0038\right)$. For the dummy-port terminations of all the lenses, we use $50 \mathrm{Ohm}$ thin film resistor in flip-chip $\mathrm{CH} 0402$ packaging [12]. Tri-focal Rotman lens model [13], [14] was used to synthesized lens's parallel plate region. The convex polygon and the expansion factor $\gamma=\sin \left(\varphi_{\max }\right) / \sin (\alpha)$ [14] of both stage lens were synthesized for $28 \mathrm{GHz}$ operation. Other design parameters for the first stage lens are: on-axis focal length $f_{1}=3 \lambda$, focal length's ratio $\beta=0.9$, sweep angle $\varphi_{\max }=40^{\circ}$, focal angle $\alpha=30^{\circ}$, and the array steering angle $\phi=40^{\circ}$. Similarly, the design parameters for the second stage lens, $f_{1}=2 \lambda, \varphi_{\max }=30^{\circ}, \alpha=30^{\circ}, \beta=0.95$ and $\theta=15^{\circ}$. We used $3 \lambda$ tapering line section to connect all the beam and array-ports to connect to $50 \mathrm{Ohm}$ microstrip transmission lines designed at $28 \mathrm{GHz}$. Note that the all the $50 \mathrm{Ohm}$ transmission lines need to phase aligned in all 6 Rotman lens PCBs.

The antenna unit cell, antenna array, first stage Rotman lens and second stage Rotman lens were all designed as separate 

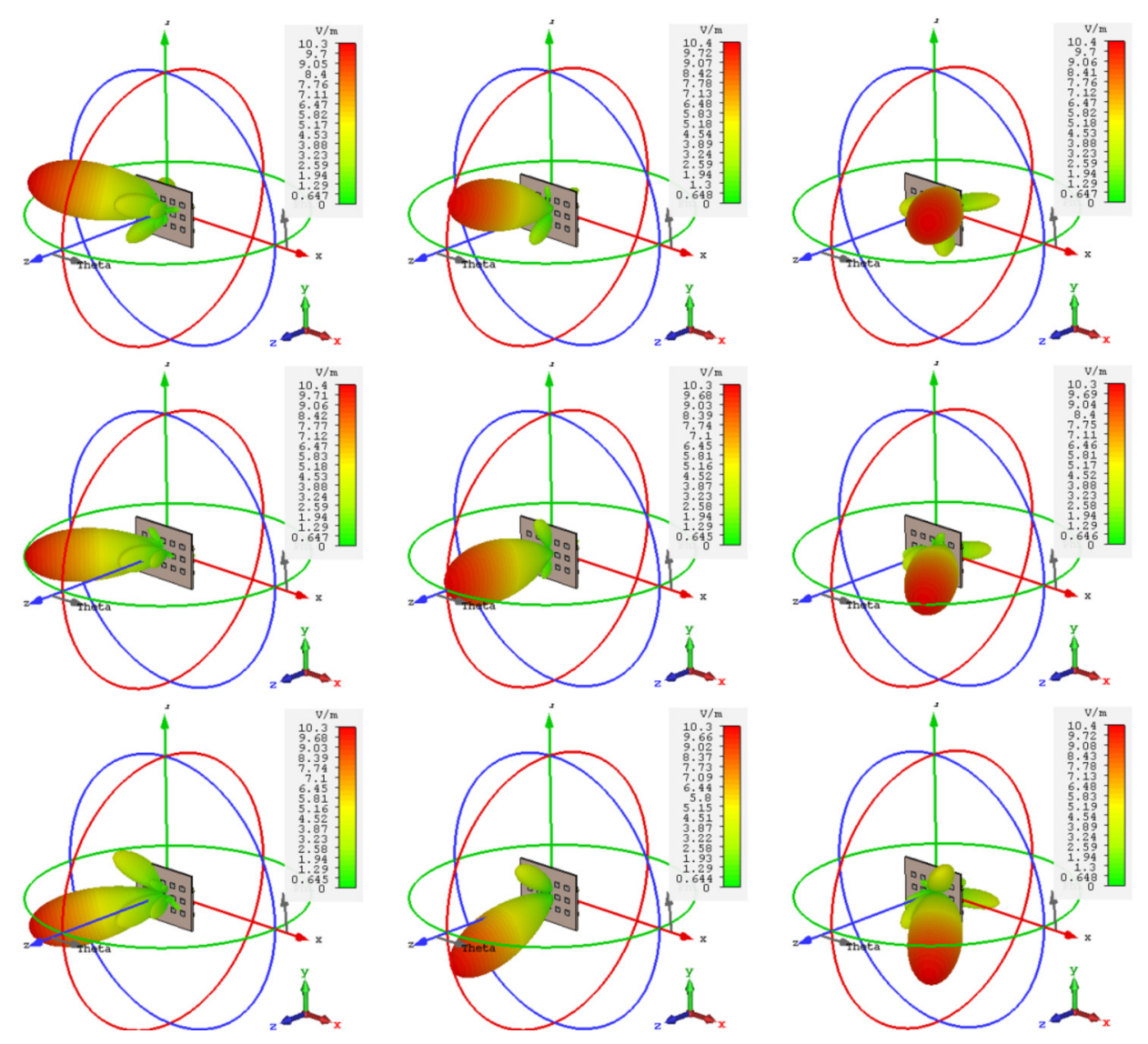

Fig. 3. Far-field patterns depicting 9 equalized beams projections by the URA when 9 beam port at the second-stage lenses were excited.

files in CST microwave studio. The antenna unit cell was simulated and optimized using the Finite Element Method (FEM) while the antenna array, the first, and the second stage Rotman lenses were simulated using the Finite-Difference Time Domain (FDTD) method. After careful investigation and ensuring the successful $28 \mathrm{GHz}$ operation of all the components separately, we combined the URA with $3 \times$ first stage lenses and $3 \times$ second stage lenses in CST Design studio. We then used the co-simulation setup to estimate the far-field beamforming performance of the URA. Final results are presented in Fig. 3 where the electric field is calculated at $1 \mathrm{~m}$ radius from the URA and is plotted in Cartesian and Spherical coordinate systems. The results clearly indicate the beam-forming capability of the proposed topology (Fig. 2 ). The design target of the final assembly along azimuth beam directions was $\left(-40^{\circ}, 0^{\circ},+40^{\circ}\right)$ and along elevation to be $\left(-15^{\circ}, 0^{\circ},+15^{\circ}\right)$. The final far-field patterns, however, depict the set of beam forming along azimuth and elevations to be $\left(-39^{\circ}, 0^{\circ},+39^{\circ}\right)$ and $\left(-16^{\circ}, 2^{\circ},+18^{\circ}\right)$ respectively. Full-wave EM simulation confirms the successful operation of the analog beamformer architecture with an accuracy up to $\pm 2^{\circ}$ in the required spatial sector. The HPBW for all the 9 beams in Fig. 3 stays within the range of $\pm 24.6^{\circ}$ to $\pm 28.1^{\circ}$ in azimuth and $\pm 29.0^{\circ}$ to $\pm 35.4^{\circ}$ in elevation zones. Although the evaluated system performance seem to achieve the requirements of MU-MIMO operation, the anomalies at the RF-front end are noticeable.

\section{HARDWARE IMPAIRMENTS AND MitigATION}

Our analysis indicated that there are three levels of hardware impairments that should be accounted for. First, within the device, second, in interconnections, and third, at the assembly level. The device level anomalies in our case arise when the theoretical operation of the lens based beamformer is unable to precisely translate to hardware. For example, consider the stag-one Rotman lens operation when port 1 is excited with a 1 Watt sinusoidal signal and electric field along the PCB (see Fig. 4). As expected, the EM energy enters the lens structure and the spherical wave front of the traveling wave couples with the array-ports, and finally travels to the $50 \mathrm{Ohm}$, transmission lines. The signal leaving the lens body will have a phase ramp required to form a beam. Out of 1 Watt power entering the beam port, almost 0.55 Watt is calculated to leave all the array ports. It is important to mention that this figure will be different for other ports of the same lens and for any port of the second-stage lens. The origin of these device level losses can further be classified into Ohmic, dielectric, radiation, return and loss due to EM energy spill-over [15]. From Fig. 4, it is evident that a significant portion of energy couples and dissipates towards one of the dummy ports, and a small portion is reflected to other beam-ports.

Some of the losses are inherent in nature and can not be mitigated. Minimizing losses in a multi-port network is itself a difficult task. Hence, mmWave RF electronics need specialized 


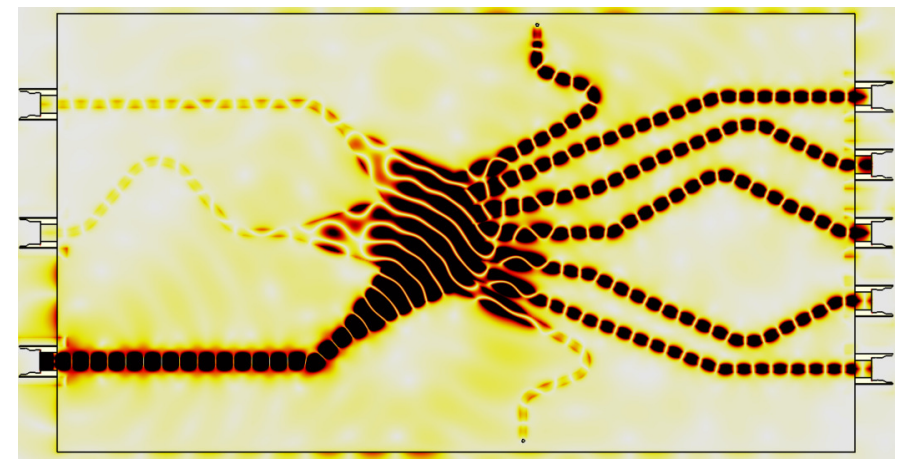

Fig. 4. Electric field distribution $200 \mu \mathrm{m}$ inside the substrate layer when the corner beam-port in first-stage Rotman lens is excited (color map is normalized).

interconnects including cables and connector which are generally lossy and costly. Assembling multiple RF blocks (like two lenses stages and URA in Fig. 2 and other similar circuits) require RF transitions, where these interconnects cannot be avoided, therefore amplifying the overall system loss. One example is given in Fig. 5, where a simple transition of a $50 \mathrm{Ohm}$ transmission line PCB with $2 \times$ SMP connectors is expected to incur $\sim 19 \%$ loss in signal power at 28 GHz. The only way around is the use of high precision connectors (like $2.92 \mathrm{~mm} K$-type) which may increase the overall implementation cost. Assembly level losses are caused due to multiple mmWave RF circuits operating in a close proximity, resulting in mutual coupling, radiation losses, and sometimes thermal discharge. The most effective way to deal with this is to isolate all the RF-paths, which sometimes is not possible in a shared space of hybrid MIMO architectures RF front-end. One possible approach is demonstrated in Fig. 6, where we relaxed sharp bends and compact transitions in phase aligned transmission lines, resulting in $\sim 30 \%$ decrease in overall radiation loss. Again, there is a trade-off between high ohmic losses in longer transmission lines, compared to high radiation and coupling losses in shorter transmission lines. We evaluated the return loss at the input SMP connections of all 9 beam ports in the second-stage lenses of the final assembly and the results are presented in Fig. 7. Return loss stays close to $-10 \mathrm{~dB}$ for all the ports with a good agreement between simulations and measurements. There is resonance shift and loss discrepancy evident from the measured return loss, which corresponds to an error of $\pm 75 \mu \mathrm{m}$ in copper trances, that we associate to tolerance of LPKR Protomate H100 milling machine and assembly anomalies. Theoretically, all RF chains in Fig. 1 are expected to see an equal power transition from the beamformer, which is not at all the case as evident from the measured data shown in Fig. 7. The narrow band behaviour of the connector from simulated results in Fig. 5 is because the microstrip line was designed to match $50 \Omega$ port impedance at $28 \mathrm{GHz}$, while the entire frequency band from 24 to 32 $\mathrm{GHz}$ was re-normalized to $50 \Omega$. Nevertheless, considering hardware impairments in mmWave RF-front is critical for an accurate link-budget, which is not a common practice in the current state of the art.

At $28 \mathrm{GHz}$, we simulate the classical double-directional

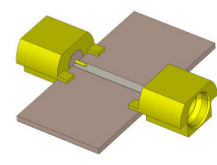

(a)

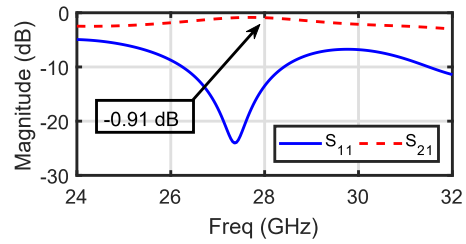

(b)
Fig. 5. (a) $28 \mathrm{GHz} 50 \mathrm{Ohm}$ microstrip transmission line with SMP mini connector at both ends, (b) Return and insertion loss predictions when ports are re-normalized to $50 \Omega$.

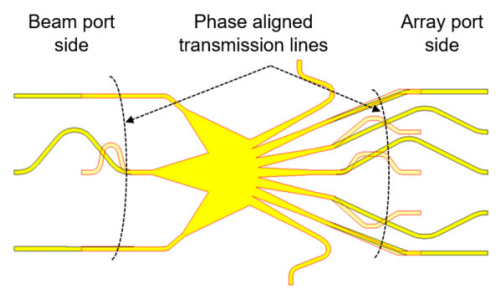

Fig. 6. First-stage lens metallic mask with longer (black contour) and shorter (red contour) transmission lines.

description far-field propagation channel model [10] with the same simulation specifications of [16] and [17] for 9 users. The net functionality of the beamformer is described by the $L \times M$ matrix:

$$
\mathbf{F}_{\mathrm{RF}}=\left[\mathbf{a}^{H}\left(\phi_{1}, \theta_{1}\right) \mathbf{a}^{H}\left(\phi_{2}, \theta_{2}\right) \ldots \mathbf{a}^{H}\left(\phi_{M}, \theta_{M}\right)\right]^{T} .
$$

Here, we used far-field patterns measured at the Queen's University Belfast anechoic chamber facility (Fig. 8 insert) for numerical evaluations instead of an ideal DFT pattern matrix. The measured data takes the focusing imperfections, spill-over and non-ideal power losses in the Rotman structure into account. Note that the remainder of the down conversion chains in (Fig. 1) are considered perfect. This assumption is valid since each of the $L$ data streams has a dedicated $\mathrm{RF}$-chain after the beamformer block. Losses per RF-chains (amplifiers, filter, mixer etc.) are considered the same for all UEs in uplink. Zero forcing (ZF) is implemented to nullify the multiuser interference, while definitions from [18] are used to compute and plot the ergodic sum spectral efficiency (see Fig. 8). An increase of $\sim 2 \mathrm{bps} / \mathrm{Hz}$ and $\sim 4 \mathrm{bps} / \mathrm{Hz}$ is expected at SNR levels of 0 and $5 \mathrm{~dB}$ when comparing the two-stage Rotman lens based architecture with a well reported single stage Rotman lens based beamformer. This is primarily because of the elevation multi-path components from elevation zone.

\section{Conclusion and Future Prospects}

Synthesis procedure, design and development of a two-stage Rotmna lens based beamformer were presented. Hardware impairments are discussed in detail when a URA and 6 stacked Rotman lenses are connected. MU-MIMO spectral efficiency of $\sim 26 \mathrm{bps} / \mathrm{Hz}$ is anticipated at an $\mathrm{SNR}=5 \mathrm{~dB}$ using the proposed beamformer architecture, showing that the approach is promising. An approximately similar trend is expected if the same design topology is re-scaled. The investigation of hardware impairments of non-ideal mmWave RF-chain 

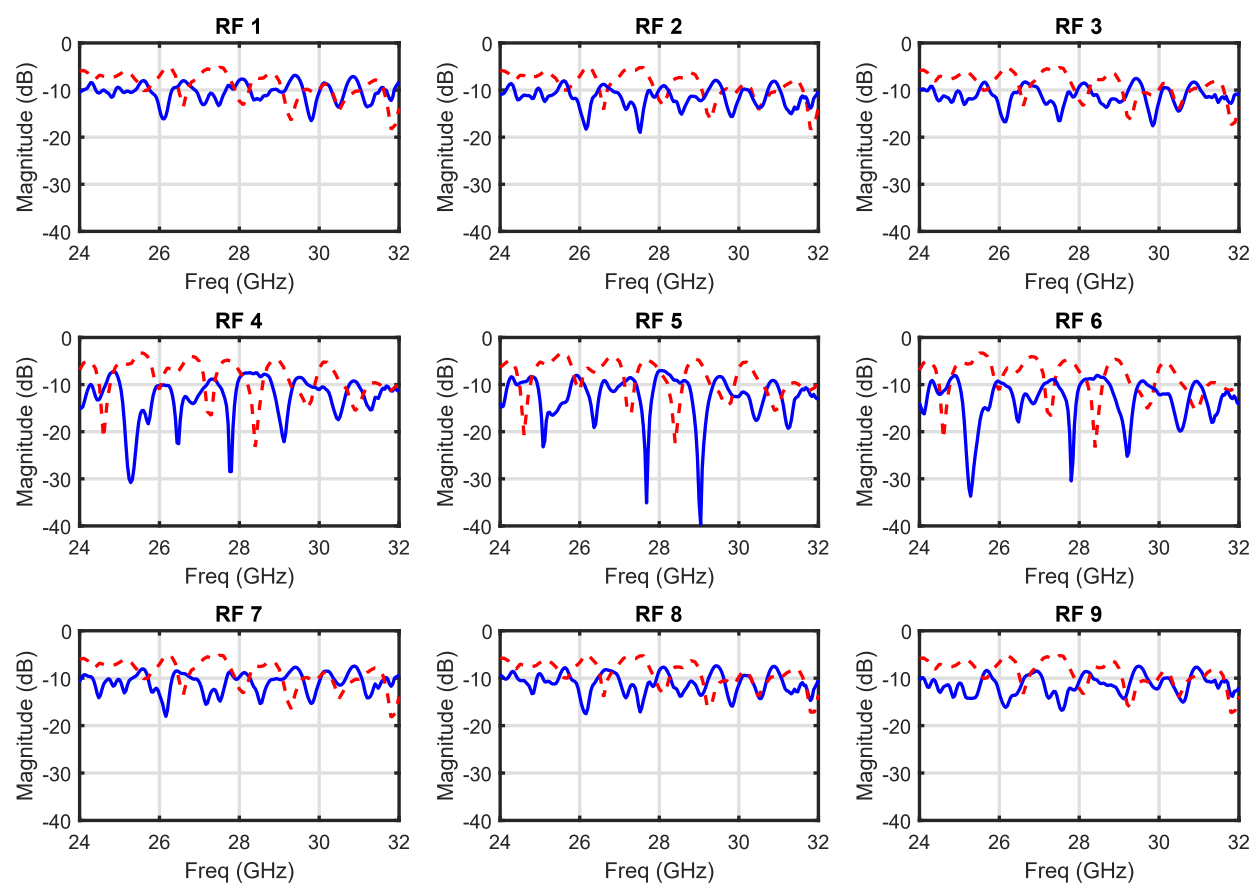

Measured - - - Simulated

Fig. 7. Comparison between simulated and measured return loss at 9 beam ports of the second-stage lenses supporting $L=9$ RF chains

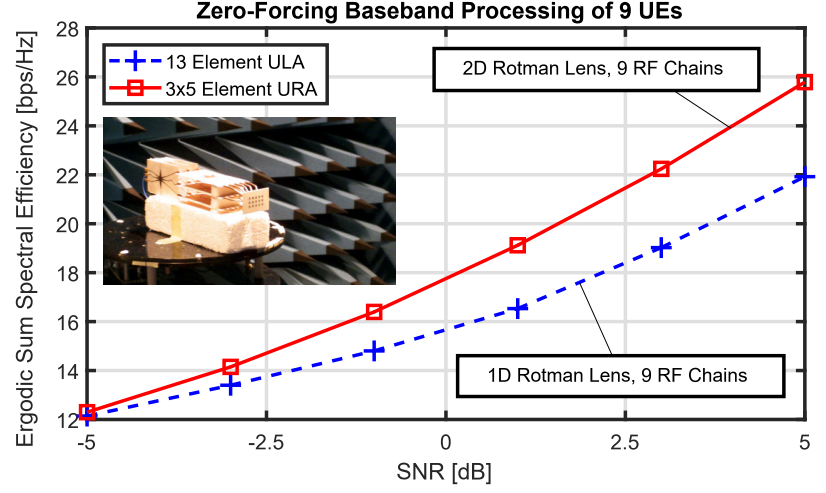

Fig. 8. Ergodic sum spectral efficiency vs. SNR (insert: Measurement setup in the NSI anechoic chamber).

components (power amplifier, low-noise amplifiers, mixer) is one of our future directions.

\section{ACKNOWLEDGEMENT}

This work is support under the grants EP/P000673/1 and EP/NO20391/1 by the UK Engineering and Physical Sciences Research Council. Thanks to H. Tataria for capacity calculations and K. Rainey for assisting in hardware realization.

\section{REFERENCES}

[1] V. Raghavan, et al., "Beamforming tradeoffs for initial UE discovery in millimeter-wave MIMO systems," IEEE J. Sel. Topics Signal Process., vol. 10, no. 3, pp. 543-559, Apr. 2016.

[2] W. Hong, et al., "Multibeam antenna technologies for 5G wireless communications," IEEE Trans. Ant. Propag., vol. 65, no. 12, pp. 62316249, Dec. 2017.

[3] Y. Zeng, L. Yang, and R. Zhang, "Multi-user millimeter-wave MIMO with full-dimensional lens antenna arrays," IEEE Trans. Wireless Commun., vol. 17, no. 4, pp. 2800-2814, Apr. 2018
[4] A. M. Sayeed and J. Brady, "Beamspace MIMO channel modeling and measurement: Methodology and results at $28 \mathrm{GHz}$," in Proc. IEEE GLOBECOM (Workshops), Dec. 2016.

[5] T. Kwon, et al., "RF lens-embedded massive MIMO systems: Fabrication issues and codebook design," IEEE Trans. Microw. Theoy Techn., vol. 64, no. 7, pp. 2256-2271, Jul. 2016.

[6] B. Yang, et al., "Digital beamforming-based massive MIMO transceiver for $5 \mathrm{G}$ millimeter-wave communications," IEEE Trans. Microw. Theory Techn., vol. 66, no. 7, pp. 3403-3418, Jul. 2018.

[7] W. Roh, et al., "Millimeter-wave beamforming as an enabling technology for $5 \mathrm{G}$ cellular communications: Theoretical feasibility and prototype results," IEEE Commun. Mag., vol. 52, no. 2, pp. 106-113, Feb. 2014.

[8] A. F. Molisch, et al., "Hybrid beamforming for massive MIMO: A survey," IEEE Commun. Mag., vol. 55, no. 9, pp. 134-141, Sep. 2017.

[9] M. A. B. Abbasi, H. Tataria, V. F. Fusco and M. Matthaiou, "Performance of a $28 \mathrm{GHz}$ two-stage Rotman lens beamformer for millimeter wave cellular systems," in Proc. IEEE EuCAP, Mar. 2019.

[10] M. Steinbauer, et al., "The double-directional radio channel," in IEEE Antennas Propag. Mag., vol. 43, no. 4, pp. 51-63, Aug. 2001.

[11] K.-L.Wong, Compact and broadband microstrip antennas. Vol. 168. John Wiley and Sons, 2004

[12] Vihay Dale RF/High Frequency Products, "High Frequency 50 GHz Thin Film Chip Resistor," CH0402 datasheet, [Revised Feb. 2018].

[13] Y. Gao, et al., "Rotman lens based hybrid analog-digital beamforming in massive MIMO systems: Array architectures, beam selection algorithms and experiments," IEEE Trans. Veh. Technol., vol. 66, no. 10, pp. 91349148, Oct. 2017.

[14] R. C. Hansen, "Design trades for Rotman lenses," IEEE Trans. Antennas Propag., vol. 39, no. 4, pp. 464-472, 1991.

[15] M. A. B. Abbasi, et al., "On the impact of spillover losses in $28 \mathrm{GHz}$ Rotman lens arrays for 5G applications," in Proc. 2018 IEEE MTT-S IMWS-5G, Oct. 2018.

[16] H. Tataria, et al., "Uplink interference analysis with RF switching for lens-based millimeter-wave systems", in Proc. IEEE ICC, May 2018 , pp. 1-7.

[17] M. A. B. Abbasi, V. F. Fusco, H. Tataria and M. Matthaiou, "Constant$\epsilon_{r}$ lens beamformer for low-complexity millimeter-wave hybrid MIMO," IEEE Trans. Microw. Theory Tech., Jun. 2019.

[18] H. Tataria, M. Matthaiou, P. J. Smith, G. C. Alexandropoulos and V F. Fusco, "Impact of RF processing and switching errors in lens-based massive MIMO systems," in Proc. 2018 IEEE SPAWC, Jun. 2018. 